\title{
Model Theory and Differential Equations
}

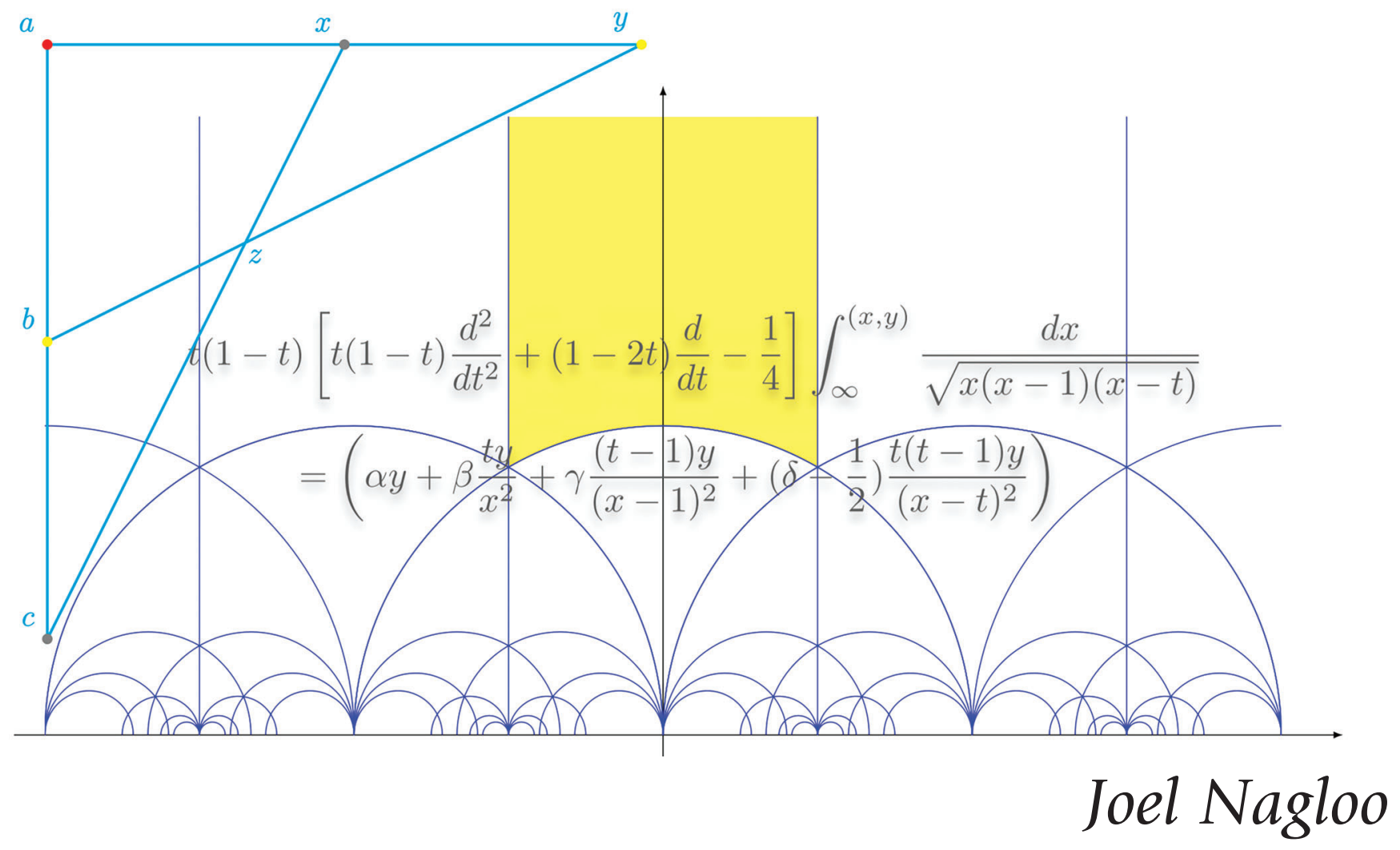

\section{Introduction}

The model-theoretic approach to the study of differential equations has a long and rich history beginning with A. Robinson [Rob59]. The theory of differentially closed fields of characteristic $0, D C F_{0}$, has been studied intensively and has played an important role in the internal development of geometric model theory. It is also behind one of the most spectacular applications of logic to number theory; namely, E. Hrushovski's celebrated proof of the function field Mordell-Lang conjecture. Furthermore, the study of the theory $D C F_{0}$ has led to substantial

Joel Nagloo is an associate professor of mathematics at CUNY Bronx Community College and Graduate Center. His email address is joe1.nag1oo@bcc .cuny.edu.

The author gratefully acknowledges support from the NSF grants DMS1700336 and DMS-1952694, and PSC-CUNY grant \#63304-00 51.

For permission to reprint this article, please contact:

reprint-permission@ams.org. development in a Galois theory for differential equations and its applications.

Nevertheless, only very recently have the techniques from model theory been used to study classical differential equations. First in the work of the author and A. Pillay on the Painleve transcendents [NP14], [NP17] and then in that of J. Freitag and T. Scanlon [FS18] on the differential equation satisfied by the modular $j$-function. More recently, in joint work with G. Casale and J. Freitag [CFN20], the author has also studied the differential equations satisfied by the Fuchsian automorphic functions and in the process proved an old claim of P. Painlevé (1895).

In this article, we give an overview of those recent applications of model theory to the study of differential equations. The focus will be on the role of the classification problem for strongly minimal sets and on results in functional transcendence. Unavoidably, many other interesting and important aspects of the interaction between model theory and differential algebra will be omitted. 


\section{Differential Algebraic Geometry}

Differential algebraic geometry, which has its origin at the beginning of the 1930s, was founded by J. Ritt and E. Kolchin. Although not widely known, it gives a general algebraic setting for the study of differential equations and the approach is similar to that of the study of polynomial equations in algebraic geometry. We will in this article focus on ordinary differential equations. Moreover, we will say a few words at the end about the setting of partial differential and difference equations. The standard reference for this section is Kolchin's book [Kol73]. All fields will be assumed to be of characteristic 0 .

Definition 1. A differential field $(K, \delta)$ is a field $K$ equipped with a derivation $\delta: K \rightarrow K$, i.e., an additive group homomorphism satisfying the Leibniz rule $\delta(x y)=x \delta(y)+$ $y \delta(x)$.

The field of constants $C_{K}$ of $K$ is defined set theoretically as $\{x \in K: \delta(x)=0\}$. We usually write $x^{\prime}$ for $\delta(x)$ and $x^{(n)}$ for $\underbrace{\delta \cdots \delta \delta}_{n}(x)$.

Example 1. $(\mathbb{C}(t), d / d t)$ is the field of rational functions over $\mathbb{C}$ in a single indeterminate, where in this case the field of constants is $\mathbb{C}$.

Associated with a differential field $(K, \delta)$ is the ring of differential polynomials $K\{\mathbf{X}\}$ in $m$ differential variables $\mathbf{X}=$ $\left(X_{1}, \ldots, X_{m}\right)$. An element of $K\{\mathbf{X}\}$ is called a differential polynomial over $K$ and is simply a regular polynomial with coefficients in $K$ but in variables $\mathbf{X}, \mathbf{X}^{\prime}, \mathbf{X}^{(2)}, \ldots$. We use here the notation $\mathbf{X}^{(n)}=\left(X_{1}^{(n)}, \ldots, X_{m}^{(n)}\right)$. If $f \in K\{\mathbf{X}\}$, then the order of $f$, denoted $\operatorname{ord}(f)$, is the largest $n$ such that for some $i, X_{i}^{(n)}$ occurs in $f$.

Example 2. $f(X)=\left(X^{\prime}\right)^{2}-4 X^{3}-t X$ is a differential polynomial in $\mathbb{C}(t)\{X\}$ and $\operatorname{ord}(f)=1$.

As one can see, if $f \in K\{\mathbf{X}\}$, then $f(\mathbf{X})=0$ is an ordinary (algebraic) differential equation. More generally, by a Kolchin closed subset of $K^{n}$, we mean the common zero set of a finite system of differential polynomial equations, i.e., a set of the form

$$
V(S)=\left\{y \in K^{n}: f(y)=0 \text { for all } f \in S\right\},
$$

where $S \subset K\{\mathbf{X}\}$ is a finite subset. The Kolchin closed sets are the basic closed sets in the Kolchin topology and are the analogues of the basic closed sets in the Zariski topology. A Kolchin constructible set is simply a boolean combination of Kolchin closed sets.

Given a differential field $(K, \delta)$, it follows that the derivation $\delta$ uniquely extends to the algebraic closure $K^{\text {alg }}$ of $K$. However, in order for Kolchin closed sets to necessarily have points whose coordinates are from the underlying field, a much stronger condition than algebraic closedness is needed.
Definition 2. A differential field $(K, \delta)$ is said to be differentially closed if for every $f, g \in K\{X\}$ such that $\operatorname{ord}(f)>$ $\operatorname{ord}(g)$, there is $y \in K$ such that $f(y)=0$ and $g(y) \neq 0$.

Differential algebraic geometry as developed by Kolchin studies Kolchin closed sets in a differentially closed field. At this point, let us mention that Kolchin closed sets can have very rich algebraic structure. Take for example the field of constants: if $K$ is differentially closed, then from Definition 2 we see that $C_{K}$ is an algebraically closed field. Less obvious is that $C_{K}$ is indeed the only algebraically closed subfield of $K$ that is given by a differential equation. Another interesting well-known example is that of a homogeneous linear differential polynomial

$$
f(X)=X^{(n)}+a_{n-1} X^{(n-1)}+\cdots+a_{1} X^{\prime}+a_{0} X, \quad a_{i} \in K .
$$

One has that the associated Kolchin closed set (in a differentially closed field $K$ ) is a vector space over $C_{K}$.

Kolchin's approach has been instrumental in the development of a Galois theory for differential equations that solidifies and extends the Picard-Vessiot theory for linear differential equations. For example, the fact that, in a differentially closed field $K$, the Galois group of a linear differential equation is a linear algebraic group defined over $C_{K}$ has been generalized in Kolchin's strongly normal theory using algebraic groups as the Galois group of so-called logarithmic equations.

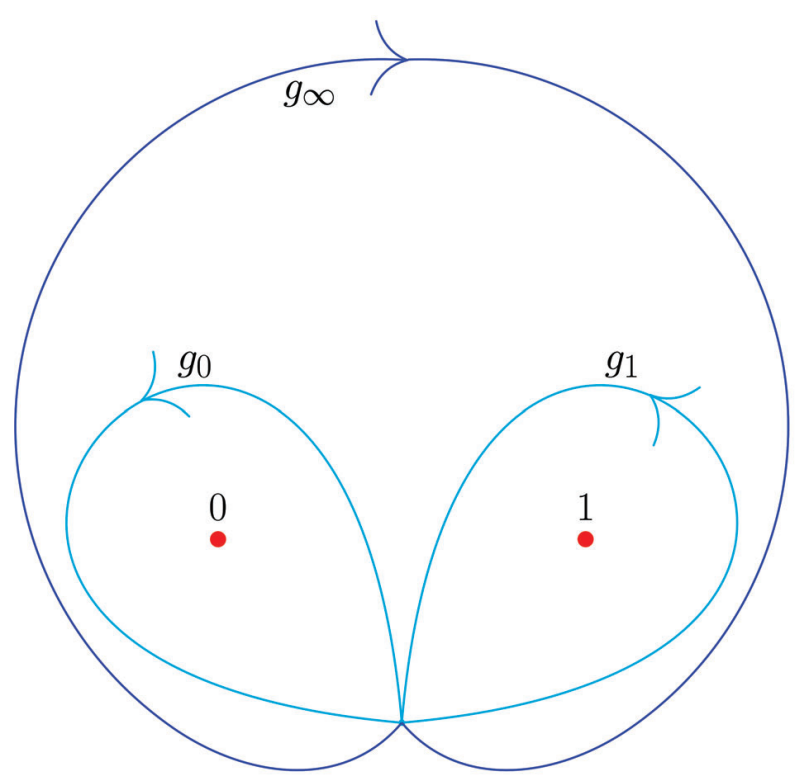

Figure 1. Loops $g_{0}, g_{1}, g_{\infty}$ in the complex plane around the singularities $0,1, \infty$ of the hypergeometric equation. The differential Galois group is the Zariski closure in $G L_{2}(\mathbb{C})$ of the monodromy group of the equation.

Kolchin's Galois theory for differential equations has helped answer questions related to the study of the structure of Kolchin constructible sets. Moreover, it can be 
argued that the point of view of Kolchin's theory coincides with that of the model theory of differentially closed fields. This is of course our approach to studying differential equations.

\section{Model Theory}

For the topics covered in this article, we recommend D. Marker's book [Mar02]. The starting point in model theory is the notion of a model of a first-order theory. Here by a first-order theory $T$ we mean a set of axioms (or more accurately first-order sentences) in a fixed language $L$. The language $L$ is simply a set of constant symbols, function symbols, and relation symbols. We assume throughout that the language is countable.

Example 3. A familiar example is $T_{G}$, the theory for groups which consist of the usual axioms for groups expressed using the language $L_{G}=(e, *)$ together with the logical symbols $=,(),, \exists$, and $\forall$.

A structure for a language $L$, or an $L$-structure for short, is a set together with interpretations for each symbol in $L$. A model of a theory $T$ is simply an $L$-structure in which the axioms are true. In Example 3, we see that both $(\mathbb{N}, 0,+)$ and $(\mathbb{Z}, 0,+)$ are $L_{G}$-structures, moreover only the latter is a model of $T_{G}$.

The notion of a (well-formed) formula extends that of an axiom, whereby free variables, that is, those not quantified upon, are allowed. Continuing with Example 3, we see that a well-formed formula with free variable $X$ is $\phi(X):=\forall Y(X * Y=Y * X)$. For a model $G$ (i.e., a group), if $C(G)$ denotes the set of elements of $G$ which satisfy the formula $\phi(X)$, then we have that $C(G)$ is the center of $G$. The center, $C(G)$, is an example of a definable set.

Definition 3. A definable set $Y \subset M^{n}$ is a set of the form

$$
Y=\left\{\mathbf{y} \in M^{n}: \phi(\mathbf{y}) \text { is true }\right\},
$$

where $\phi$ is a formula in $L$ with $n$ free variables.

Remark 1. For any subset $A \subset M$ of a model, one can extend the language $L$ by adding a constant symbol for each element $a \in A$. One usually denotes the new language obtained by $L_{A}$. If in Definition 3 one replaces $L$ by $L_{A}$ for some $A \subset M$, then one obtains the definition of an $A$-definable set or more precisely a definable set with $p a$ rameters from the set $A$.

For a fixed theory $T$ a major goal of model theory is to study all definable sets in some/any model of $T$. This of course would be hopeless unless one could identify classes of structures where there is some control over the definable sets. In model theory, this leads to the distinction between "tame" and "wild" structures or theories. In this article we discuss two notions of tameness, namely quantifier elimination and $\omega$-stability. There are many more natural

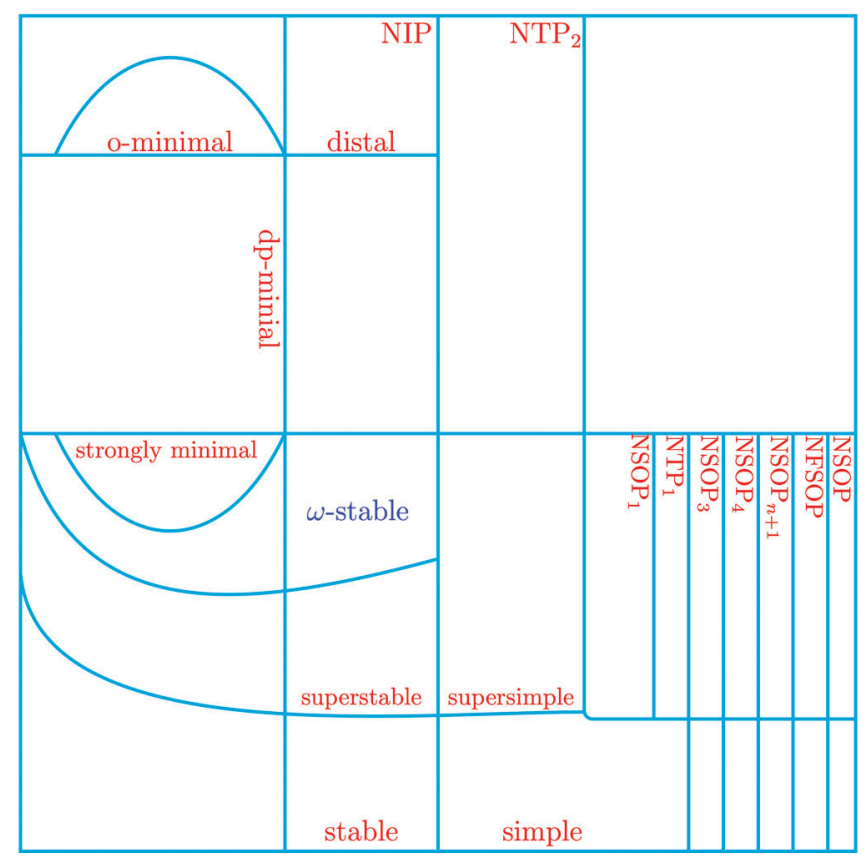

Figure 2. The model theory universe as described at forkinganddividing. com. In the sea of theories, an $\omega$-stable theory is ideally placed in the center left of the natural "tame/wild" divide.

"tame" versus "wild" distinctions and some are illustrated in Figure 2.

A theory $T$ is said to have quantifier elimination if for every formula $\phi(\mathbf{X})$ there is a quantifier-free formula $\psi(\mathbf{X})$ such that the two define the same definable set. It hence follows that for theories with quantifier elimination the definable sets are defined using "simple" formulas.

A theory $T$ is $\omega$-stable if every definable set $X$ can be given an intrinsic ordinal-valued dimension called the Morley rank, denoted by $R M(X)$. In rough terms, the inductive definition is as follows: $R M(X)=0$ if $X$ is finite, and $R M(X) \geq \alpha+1$ if there are pairwise disjoint definable subsets $X_{i}$ of $X$ for $i=1,2, \ldots$ such that each $R M(X) \geq \alpha$ for all $i<\omega$ (one extends the definition naturally to limit ordinals). We set $R M(X)=\alpha$ if $R M(X) \geq \alpha$ but not $\geq \alpha+1$. Using this rank, one can define in $T$ a good notion of independence and dimension analogous to the notion of linear independence and basis in the study of vector space.

The theory of algebraically closed fields of characteristic zero, $A C F_{0}$, with the obvious axioms given in the language of rings $L_{R}=(+,-, \cdot, 0,1)$ has both quantifier elimination and is $\omega$-stable. In this setting quantifier elimination is equivalent to the Chevalley-Tarski theorem that over an algebraically closed field the projection of a constructible set is constructible. The Morley rank of a definable set (so a constructible set) corresponds to the transcendence degree of a generic point, while the independence notion is equivalent to algebraic independence. 


\section{The Theory $D C F_{0}$}

Let us bring together the ideas of the first two sections. We refer the reader to [MMP96] for historical background and additional details. In the context of differentially closed fields, the relevant language is $L_{\delta}=(+,-, \cdot, \delta, 0,1)$, the language of differential rings, and we denote by $D F_{0}$ the theory of differential fields of characteristic zero. The axioms of $D F_{0}$ consist of the axioms for fields and the axioms for the derivation $\delta$.

Now, for each $n, d_{1}$, and $d_{2} \in \mathbb{N}$, one can write down an axiom (in $L_{\delta}$ ) that asserts that if $f$ is a differential polynomial of order $n$ and degree at most $d_{1}$ and $g$ is a nonzero differential polynomial of order less than $n$ and degree at most $d_{2}$, then there is a solution to $f(X)=0$ and $g(X) \neq 0$. The theory obtained by adding to $D F_{0}$ all these axioms is called the theory of differentially closed fields of characteristic $0, D C F_{0}$. This theory sits on the tame side of many of the most important dividing lines in model theory as shown by Blum [Blu69].

Theorem 1. The theory $D C F_{0}$ eliminates quantifiers and is $\omega$-stable.

For the remainder of the article $\mathcal{U}$ will denote a saturated $^{1}$ model of $D C F_{0}$.

Quantifier elimination means that a definable set $Y \subseteq$ $\mathcal{U}^{n}$, definable over a differential subfield $K$ of $\mathcal{U}$, is nothing more than a Kolchin constructible set over $K$. On the other hand, as discussed above, $\omega$-stability means (among other things) that any definable set has a well-defined ordinal-valued Morley rank. The independence notion in $\mathcal{U}$ is as follows: $\mathbf{a}$ is independent from $\mathbf{b}$ over $K$ if $K\langle\mathbf{a}\rangle$ is algebraically disjoint from $K\langle\mathbf{b}\rangle$ over $K$. Here $K\langle\mathbf{a}\rangle=$ $K\left(\mathbf{a}, \mathbf{a}^{\prime}, \mathbf{a}^{(2)}, \ldots\right)$ denotes the differential field generated by a over $K$.

Along with the Morley rank, we also have another invariant for definable sets called the order. For $\mathbf{a} \in \mathcal{U}^{n}$ and $K<\mathcal{U}$, we define $\operatorname{ord}(\mathbf{a} / K)$ to be the transcendence degree of the field $K\langle\mathbf{a}\rangle$ over $K$. If $Y \subseteq \mathcal{U}^{n}$ is definable over $K$, we define $\operatorname{ord}(Y)=\sup \{\operatorname{ord}(\mathbf{a} / K): \mathbf{a} \in Y\}$. One can show that $R M(Y)$ is always less than or equal to $\operatorname{ord}(Y)$. Furthermore, $R M(Y)<\omega$ if and only if $\operatorname{ord}(Y)<\omega$. We will later see examples of Kolchin closed sets for which the Morley rank is strictly less than the order.

Definition 4. Let $Y \subseteq \mathcal{U}^{n}$ be a definable set.

1. $Y$ is said to be finite dimensional (or rank) if it has finite order, i.e., $\operatorname{ord}(Y)<\omega$.

2. $Y$ is said to be strongly minimal if it is infinite and for every definable subset $Z \subseteq Y$, either $Z$ or $Y \backslash Z$ is finite.

\footnotetext{
${ }^{1}$ Saturation is a notion of "largeness" which mimics the idea that an algebraically closed field of uncountable transcendence degree over the prime field is large/rich.
}

If $Y$ is strongly minimal, then it has Morley rank one. Strongly minimal sets determine, in a precise manner (not to be discussed in this article), the structure of all finitedimensional definable sets. This fact, which follows from very general model-theoretic considerations, holds in any $\omega$-stable theory and is obtained in part using the robust notion of independence.

Notice that if $Y$ is a definable set with $\operatorname{ord}(Y)=n$, then $Y$ is strongly minimal if and only if $Y$ cannot be written as the disjoint union of definable sets of order $n$, and for any differential field $K$ over which $Y$ is defined and element $y \in Y$, then $\operatorname{tr} \cdot \operatorname{deg}(K\langle y\rangle / K)=0$ or $n$.

Example 4. The field of constants $C_{\mathcal{U}}$ is strongly minimal.

Example 5. If $f$ is an absolutely irreducible polynomial over $U$ in two variables, then the subset $Y$ of $U$ defined by $f\left(y, y^{\prime}\right)=0$ is strongly minimal, of order 1 .

It is quite a difficult task to show that the set defined by a given differential equation is strongly minimal. Indeed, except for limited or special cases, no general tools are available. For example, we refer the reader to Section 5.17 of [MMP96] for the (tedious) calculations involved in showing that the subset of $\mathcal{U}$ defined by $\left\{y y^{\prime \prime}=y^{\prime}, y^{\prime} \neq 0\right\}$ is strongly minimal, of order 2 .

Nevertheless, the goal of understanding all definable sets in $D C F_{0}$ goes through a complete understanding of the strongly minimal sets. A considerable amount of work, beginning in the 1990s, has been devoted to just that. The deepest result in that direction, due to E. Hrushovski and Z. Sokolovic [HS94], concerns the classification of strongly minimal sets that have "nontrivial" structures.

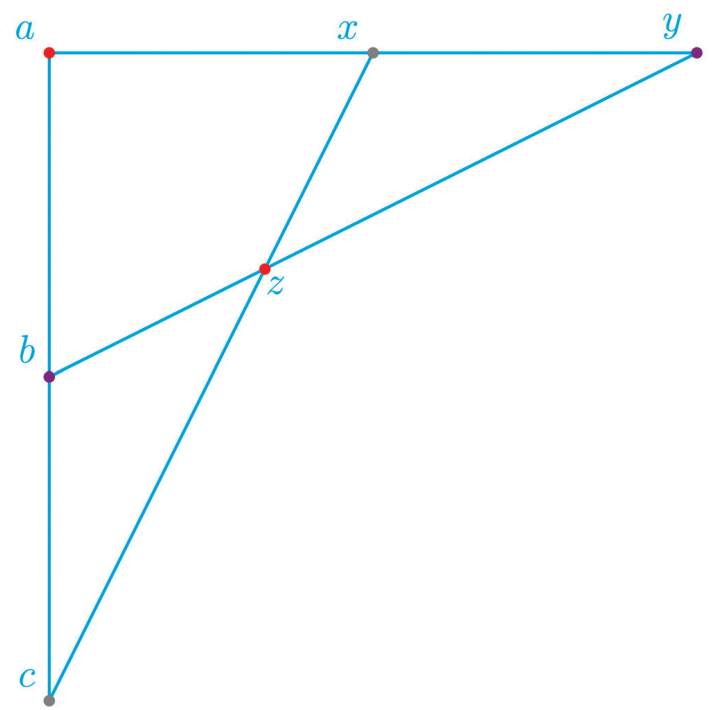

Figure 3. Presence of a definable group: in a nongeometrically trivial strongly minimal set one can find a group configuration. Each point has (Morley) rank 1, each line rank 2, and any three noncollinear points are independent. 
Definition 5. Let $Y$ be a strongly minimal set defined over a differential field $K$. Then $Y$ is said to be geometrically trivial if for any $y, y_{1}, \ldots, y_{n} \in Y$ if $y \in K\left\langle y_{1}, \ldots, y_{n}\right\rangle^{\text {alg }}$, then there is $1 \leq i \leq n$ such that $y \in K\left\langle y_{i}\right\rangle^{\text {alg }}$.

In essence, a geometrically trivial set can have at most a "binary" structure. The field of constants $C_{\mathcal{u}}$ is not geometrically trivial. The same is true of definable groups (i.e., definable sets equipped with definable group structures).

The work of Hrushovski and Sokolovic did not attempt to classify geometrically trivial strongly minimal sets. On the other hand, a key step in their work and which builds on those of A. Buium [Bui92] was the identification of some "exotic" differential algebraic groups (i.e., definable groups where the underlying definable set is Kolchin closed not Zariski closed).

Theorem 2. Let $A$ be an abelian variety over $\mathcal{U}$. We identify $A$ with its set $A(\mathcal{U})$ of $\mathcal{U}$-points. Let $A^{\sharp}$ be the Kolchin closure of the torsion subgroup of $A$. Then:

1. $A^{\sharp}$ is a differential algebraic group and is Zariski dense in $A$.

2. If $A$ is a simple abelian variety that does not descend to $C_{\mathcal{U}}$, then $A^{\sharp}$ is strongly minimal.

The group $A^{\sharp}$ is called the Manin kernel of $A$. One remarkable property of $A^{\sharp}$ is that every definable subset of it is a finite Boolean combination of cosets of definable subgroups. The result of Hrushovski and Sokolovic is that, up to equivalence, the field of constants $C_{u}$ and the groups $A^{\sharp}$ cover all the nongeometrically trivial examples!

Theorem 3 (The trichotomy theorem). If $Y \in \mathcal{U}^{n}$ is strongly minimal, then exactly one of the following hold:

1. $Y$ is geometrically trivial, or

2. (Group-like) $Y$ is nonorthogonal to the Manin kernel $A^{\sharp}$ of some simple abelian variety $A$ that does not descend to $C_{\mathcal{U}}$, or

3. (Field-like) $Y$ is nonorthogonal to the field of constants $C_{\mathfrak{u}}$.

We say that $Y$ and $Z$ (both strongly minimal) are nonorthogonal if there is some infinite definable relation $R \subset Y \times Z$ such that $\pi_{Y \uparrow R}$ and $\pi_{Z \uparrow R}$ are finite-to-one functions. Here $\pi_{Y}: Y \times Z \rightarrow Y$ and $\pi_{Z}: Y \times Z \rightarrow Z$ denote the projections to $Y$ and $Z$, respectively. It is not hard to see that nonorthogonality is indeed an equivalence relation on strongly minimal sets. Furthermore, if $Y$ and $Z$ are nonorthogonal strongly minimal sets, then $\operatorname{ord}(Y)=\operatorname{ord}(Z)$.

The work of Hrushovski and Sokolovic was never published. Moreover, an alternate proof of the characterization of the field-like strongly minimal sets-a key stephas appeared in the work of A. Pillay and M. Zeigler [PZ03].
A good summary of the proof of Theorem 3 can be found in [NP17, Section 2.1].

There are other interesting and important consequences of the trichotomy theorem that are not apparent but worth mentioning. Firstly, if $A^{\sharp}$ is the Manin kernel of a simple abelian variety $A$ that does not descend to $C_{\mathcal{u}}$, then $\operatorname{ord}\left(A^{\sharp}\right) \geq 2$. Hence, strongly minimal sets of order 1 are either geometrically trivial or nonorthogonal to $C_{\mathcal{U}}$. Secondly, strongly minimal sets that are defined over $C_{u}$ and of order $\geq 2$ are geometrically trivial! This surprising fact was somewhat forgotten for a while but now plays a crucial role in some of the applications of the theory to functional transcendence as we shall see in the next section.

Finally, it is worth mentioning that strong minimality is closely related to Painlevés notion of irreducibility of differential equations. Roughly speaking, a differential equation is irreducible if none of it solutions are "known" special functions. Establishing irreducibility, which goes through establishing strong minimality, has been part of long-standing open conjectures in the theory of nonlinear special function.

\section{Trivial Pursuits and Applications}

As we have seen, the trichotomy theorem, which gives a very general classification theorem for strongly minimal sets, has nothing to say about geometrically trivial strongly minimal sets. Understanding these strongly minimal sets, or trivial pursuits (as coined by J. Baldwin and L. Harrington), is one of the most important open problems in the study of $D C F_{0}$. But to this date very little progress has been made.

For a while it was conjectured that all geometrically trivial strongly minimal sets would have no (or very little) structure: for any element $y$ of a trivial strongly minimal set $Y$ only finitely many other elements of $Y$ are interalgebraic with $y$. More precisely,

Definition 6. Let $Y$ be a strongly minimal set defined over a differential field $K$. Then $Y$ is said to be $\omega$-categorical if for any tuple $\mathbf{b}$ from $\mathcal{U}$, the set $K\langle\mathbf{b}\rangle^{\text {alg }} \cap Y$ is finite.

If a strongly minimal set is $\omega$-categorical, then it is geometrically trivial. A beautiful result of E. Hrushovski [Hru95] is that the converse holds for order 1 strongly minimal sets (cf. [Pil02, Cor 1.82] and [FM17] for a generalization).

Theorem 4. Let $Y \subset \mathcal{U}^{n}$ be an order 1 geometrically trivial strongly minimal set. Then $Y$ is $\omega$-categorical.

This result of Hrushovski gave rise to a conjecture about geometrically trivial strongly minimal sets of arbitrary order: in differentially closed fields, every geometrically trivial strongly minimal set is $\omega$-categorical. This was proven to be false at this level of generality in [FS18] using the order 3 
differential equation satisfied by the modular $j$-function (see below). The following interesting question remains.

Question 1. Are all order 2 geometrically trivial strongly minimal sets $\omega$-categorical?

At this point, let us mention that if a strongly minimal set $Y$ has $\operatorname{ord}(Y)=n$ and is defined over $K$, then $\omega$-categoricity can be translated to the following strong transcendence statement: there is an $m \in \mathbb{N}$ such that if $y_{1}, \ldots, y_{k} \in Y$ are distinct and satisfy $\operatorname{tr} \cdot \operatorname{deg}\left(K\left\langle y_{1}, \ldots, y_{k}\right\rangle / k\right)=n k$, then for any other $y \in Y$, except for at most $m k$, we have that $\operatorname{tr} \cdot \operatorname{deg}\left(K\left\langle y_{1}, \ldots, y_{k}, y\right\rangle / k\right)=n(k+1)$. It follows that establishing strong minimality, geometric triviality, and $\omega$ categoricity can be seen as part of a strategy to tackle number-theoretic/functional transcendence type results for the solutions of the differential equations. As such, a positive answer to the above question is of great interest. We will now illustrate this by looking at several recent applications of the model-theoretic approach, in particular the trivial pursuits, to some classical differential equations.

The generic Painlevé transcendents. The Painlevé equations are second-order ordinary differential equations and come in six families $P_{I}-P_{V I}$, where $P_{I}$ consists of the single equation

$$
\frac{d^{2} y}{d t^{2}}=6 y^{2}+t
$$

and $P_{I I}-P_{V I}$ come with some complex parameters. They were isolated in the early part of the 20th century by P. Painlevé, with refinements by B. Gambier and R. Fuchs, as those ODE's of the form $y^{\prime \prime}=f\left(y, y^{\prime}, t\right)$ (where $f$ is rational over $\mathbb{C}$ ) which have the Painlevé property: any local analytic solution extends to a meromorphic solution on the universal cover of $P^{1}(\mathbb{C}) \backslash S$, where $S$ is the finite set of singularities of the equation. The equations have arisen in a variety of important physical applications including, for example, statistical mechanics, general relativity, and fiber optics.

Example 6. The second Painlevé equation $P_{I I}(\alpha)$ is given by

$$
\frac{d^{2} y}{d t^{2}}=2 y^{3}+t y+\alpha
$$

where $\alpha \in \mathbb{C}$. The equation appears quite prevalently in random matrix theory (cf. [FW15]).

Painleve believed that, at least for general values of the parameters, the set defined by the equations would be strongly minimal. This was proven to be true in a series of papers by K. Okamoto, K. Nishioka, M. Noumi, H. Umemura, and H. Watanabe (cf. [Oka99] for a survey). In particular, the first Painlevé equation is strongly minimal and in the case of the second Painleve equation, they proved that $P_{I I}(\alpha)$ is strongly minimal if and only if $\alpha \notin \frac{1}{2}+\mathbb{Z}$. By a generic Painlevé equation we mean one equation among the family $P_{I}-P_{V I}$, such that all the corresponding complex parameters are transcendental and algebraically independent over $\mathbb{Q}$. So $P_{I I}(\pi)$ is a generic equation. The works of Watanabe and others hence give that all the generic Painlevé equations are strongly minimal. They left wide open the question of the fine structure of the definable sets. We now have a full answer.

Theorem 5. Suppose $y_{1}, \ldots, y_{n}$ are distinct solutions of one of the generic Painleve equations. Then $y_{1}, y_{1}^{\prime}, \ldots, y_{n}, y_{n}^{\prime}$ are algebraically independent over $\mathbb{C}(t)$, i.e.,

$$
\operatorname{tr} . \operatorname{deg}\left(\mathbb{C}(t)\left(y_{1}, y_{1}^{\prime}, \ldots, y_{n}, y_{n}^{\prime}\right) / \mathbb{C}(t)\right)=2 n .
$$

In particular the generic Painlevé equations are all $\omega$ categorical. K. Nishioka [Nis04] proved the result for $P_{I}$ using differential algebra. However his calculations and techniques do not seem to generalize to the other equations. The author, in [Nag20] and before that in joint work with Pillay in [NP14], proved the result for all the other equations using model theory. The proofs rely heavily on earlier work [NP17] in which the trichotomy is used to show that the generic Painlevé equations are all geometrically trivial.

The model-theoretic approach has also allowed us to show that the generic equations from most distinct Painleve families are orthogonal. Work is currently underway towards obtaining a full classification of algebraic relations between solutions of the Painlevé equations. As of now, except for the second Painleve equation (where for example the author showed geometrically that triviality holds if and only if $\alpha \notin \frac{1}{2}+\mathbb{Z}$ ), the study of the nongeneric Painlevé equations is wide open. The following is an example of the most basic question one would like to answer.

Question 2. For which values of the parameters of a fixed Painlevé equation is it true that if $y_{1}, \ldots, y_{n}$ are distinct solutions (not in $\mathbb{C}(t)^{\text {alg }}$ ), then

$$
\operatorname{tr} \cdot \operatorname{deg}\left(\mathbb{C}(t)\left(y_{1}, y_{1}^{\prime}, \ldots, y_{n}, y_{n}^{\prime}\right) / \mathbb{C}(t)\right)=2 n ?
$$

Fuchshian automorphic functions. We now consider the most natural generalizations of the trigonometric and elliptic functions (i.e., the periodic functions).

Let $\Gamma \subset P S L_{2}(\mathbb{R})$ be a Fuchsian group, that is, assume that $\Gamma$ is a discrete subgroup of $P S L_{2}(\mathbb{R})$. A point $\tau \in \mathbb{H} \cup \mathbf{P}^{1}(\mathbb{R})$ is said to be a cusp if its stabilizer group $\Gamma_{\tau}=\{g \in \Gamma: g \cdot \tau=\tau\}$ has infinite order. We also assume throughout that $\Gamma$ is of first kind (i.e., its limit set is $\mathbf{P}^{1}(\mathbb{R})$ ) and of genus zero (i.e., $\Gamma \backslash \mathbb{M}$ can be compactified to a compact Riemann surface of genus 0 ). An automorphic function $f$ for $\Gamma$ is a function on the complex upper half plane $\mathbb{t}$, 


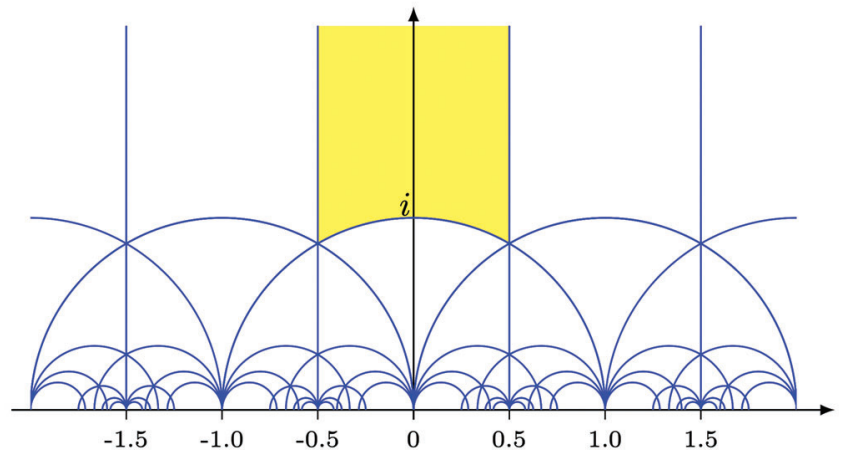

Figure 4. The fundamental domain for the action of a Fuchsian group of the first kind-here $\mathrm{PSL}_{2}(\mathbb{Z})$ - has a finite number of generators and is of finite volume.

such that ${ }^{2}$

$$
f(g \cdot \tau)=f(\tau) \quad \text { for all } g \in \Gamma \text { and } \tau \in \mathbb{H},
$$

and such that $f$ is meromorphic at every cusp of $\Gamma$. The collection $\mathcal{A}_{0}(\Gamma)$ of all automorphic functions for $\Gamma$ is a field and is generated (over $\mathbb{C}$ ) by some automorphic function called an hauptmodul or uniformizer for $\Gamma$. We will denote by $j_{\Gamma}(t)$ one such fixed hauptmodul.

It is a classical fact that $j_{\Gamma}(t)$ satisfies a third-order ordinary differential equation of Schwarzian type,

$$
S_{t}(y)+\left(y^{\prime}\right)^{2} R_{j_{\Gamma}}(y)=0 .
$$

Here $S_{t}(y)=\left(\frac{y^{\prime \prime}}{y^{\prime}}\right)^{\prime}-\frac{1}{2}\left(\frac{y^{\prime \prime}}{y^{\prime}}\right)^{2}$ denotes the Schwarzian derivative $\left({ }^{\prime}=\frac{d}{d t}\right)$ and

$$
R_{j_{\Gamma}}(y)=\frac{1}{2} \sum_{i=1}^{r} \frac{1-\alpha_{i}^{-2}}{\left(y-a_{i}\right)^{2}}+\sum_{i=1}^{r} \frac{\beta_{i}}{y-a_{i}}
$$

with $a_{1}, \ldots, a_{n}$ and $\beta_{1}, \ldots, \beta_{n}$ real numbers depending on $\Gamma$ and $j_{\Gamma}$. Every solution in $\mathcal{U}$ of the Schwarzian equation $(\star)$ can be taken to be of the form $j_{\Gamma}(g \cdot t)$ for some $g \in G L_{2}(\mathbb{C})$.

Example 7. If $\Gamma=P S L_{2}(\mathbb{Z})$, then the classical modular $j$-function

$$
j(\tau)=\frac{1}{q}+744+196884 q+21493760 q^{2}+\cdots,
$$

where $q=e^{2 \pi i \tau}$, is an hauptmodul. In this case the differential equation is given with

$$
R_{j}(y)=\frac{y^{2}-1968 y+2654208}{y^{2}(y-1728)^{2}} .
$$

P. Painlevé in 1895 again claimed that the set defined by equation $(\star)$ would be strongly minimal. K. Nishioka proved that the hauptmodul $j_{\Gamma}$ does not satisfy any algebraic differential equation of order two or less over $\mathbb{C}\left(t, e^{\lambda t}\right)$ for any $\lambda \in \mathbb{C}$. He also obtained a very weak form of

\footnotetext{
${ }^{2}$ Throughout $g \cdot \tau$ will denote the action of an element of $G L_{2}(\mathbb{C})$ by linear fractional transformation.
}

the Painleve assertion in the case of triangle groups. The first real progress was made by J. Freitag and T. Scanlon [FS18] in their work on the modular $j$-function (they did not know of Painlevé's claim then).

Theorem 6. Let $\Gamma=P S L_{2}(\mathbb{Z})$. Then the set defined by the Schwarzian equation $(\star)$ is strongly minimal and geometrically trivial but not $\omega$-categorical.

Their proof relies on a deep functional transcendence result of J. Pila [Pil13] called the modular Ax-LindemannWeierstrass theorem with derivatives (see below).

Remark 2. Granted that strong minimality holds, it is rather unsurprising that the definable set in the case of the $j$-function is not $\omega$-categorical. Indeed, for each $n \in \mathbb{N}$ we have the classical modular polynomials $\Phi_{n}(X, Y) \in \mathbb{Z}[X, Y]$ that relate solutions of the equation for $j$ : if $g_{1}$ and $g_{2}$ are in the same coset of $G L_{2}(\mathbb{Q})$, then $\Phi_{n}\left(j\left(g_{1} \cdot t\right), j\left(g_{1} \cdot t\right)\right)=0$ for some $n$.

For a while the result of Freitag and Scanlon seemed to have shut the door on a possible classification of geometrically trivial strongly minimal sets. However, it turned out that studying the Schwarzian equation $(\star)$ in its full generality has allowed us to place the case $\Gamma=P S L_{2}(\mathbb{Z})$ in context. A natural and key question is the following: is there a way to explain the existence of the modular polynomials? The answer is again very classical and is brought to light through the notion of commensurability.

Recall that two subgroups $G$ and $H$ of $P S L_{2}(\mathbb{R})$ are commensurable, denoted by $G \sim H$, if their intersection $G \cap H$ has finite index in both $G$ and $H$. For a Fuchsian group $\Gamma$, let $\operatorname{Comm}(\Gamma)$ be the commensurator of $\Gamma$, namely

$$
\operatorname{Comm}(\Gamma)=\left\{g \in P S L_{2}(\mathbb{R}): g \Gamma g^{-1} \sim \Gamma\right\} .
$$

If $g \in \operatorname{Comm}(\Gamma) \backslash \Gamma$, then one has that the intersection $\Gamma_{g}=g \Gamma g^{-1} \cap \Gamma$ is a Fuchsian group of first kind and with the same set of cusps as $\Gamma$. But the functions $j_{\Gamma}(t)$ and $j_{\Gamma}\left(g^{-1} t\right)$ are respective uniformizers for $\Gamma$ and $g \Gamma g^{-1}$. It follows that they also are automorphic functions for $\Gamma_{g}$. The work of $\mathrm{H}$. Poincaré gives that any two automorphic functions for a Fuchsian group are algebraically dependent over $\mathbb{C}$. So there is a polynomial $\Phi_{g} \in \mathbb{C}[X, Y]$, such that $\Phi_{g}\left(j_{\Gamma}(t), j_{\Gamma}(g \cdot t)\right)=0$. Such a polynomial is called a $\Gamma-$ special polynomial.

So if $\Gamma$ has infinite index in $\operatorname{Comm}(\Gamma)$, then there are infinitely many $\Gamma$-special polynomials. In particular, if one can prove strong minimality, then non- $\omega$-categoricity would follow. It turns out that groups $\Gamma$ having this "infinite index" property are well known in group theory.

Let $F$ be a totally real number field, and let $A$ be a quaternion algebra over $F$ that is ramified at exactly one infinite place. Let $\rho$ be the unique embedding of $A$ into $M_{2}(\mathbb{R})$, and let $\mathcal{O}$ be an order in $A$. The image $\rho\left(\mathcal{O}^{1}\right)$ of the normone group of $\mathcal{O}$ under $\rho$ is a discrete subgroup of $S L_{2}(\mathbb{R})$. 
We denote by $\Gamma(A, \mathcal{O})$ the Fuchsian group obtained under the projection in $P S L_{2}(\mathbb{R})$ of the group $\rho\left(\mathcal{O}^{1}\right)$.

Definition 7. A Fuchsian group $\Gamma$ is said to be arithmetic if it is commensurable with a group of the form $\Gamma(A, \mathcal{O})$.

The modular group $\mathrm{PSL}_{2}(\mathbb{Z})$ and its finite index subgroups are the most well-known examples of arithmetic groups. We have the following deep result of G. Margulis.

Theorem 7. The group $\Gamma$ is arithmetic if and only if it has infinite index in $\operatorname{Comm}(\Gamma)$ and so there are infinitely many $\Gamma$ special polynomials.

The work of the author with G. Casale and J. Frietag [CFN20] completely proves Painlevé's claim and provides a striking connection between categoricity and arithmeticity.

Theorem 8. Let $\Gamma$ be a Fuchsian group of first kind and genus zero, and let $X_{\Gamma}$ be the set defined by the Schwarzian equation $(\star)$. Then:

1. $X_{\Gamma}$ is strongly minimal and (so) geometrically trivial.

2. $X_{\Gamma}$ is $\omega$-categorical if and only if $\Gamma$ is nonarithmetic.

The techniques in the proof of Theorem 8 rely on differential Galois theory, monodromy of linear differential equations, the study of algebraic and Liouvillian solutions, differential algebraic work of Nishioka towards the Painleve irreducibility of certain Schwarzian equations, and considerable machinery from the model theory of differentially closed fields. The following question can be seen as the next major challenge in the classification of geometrically trivial strongly minimal sets in differentially closed fields.

Question 3. In $D C F_{0}$, does every non- $\omega$-categorical strongly minimal set arise from an arithmetic Fuchsian group in this way?

Finally, let us mention that the above work on fully classifying the structure of the definable sets associated with the Schwarzian equation $(\star)$ has been used in [CFN20] to give a proof of the Ax-Lindemann-Weierstrass theorem with derivatives for $\Gamma$ : let $V \subset \mathbb{A}^{n}$ be an irreducible algebraic variety defined over $\mathbb{C}$ such that $V(\mathbb{C}) \cap \mathbb{Q}^{n} \neq \emptyset$ and $V$ projects dominantly to each of its coordinates (each coordinate function is nonconstant). Let $t_{1}, \ldots, t_{n}$ be the functions on $V$ induced by the canonical coordinate functions on $\mathbb{A}^{n}$. We say that $t_{1}, \ldots, t_{n}$ are $\Gamma$-geodesically independent if there are no relations of the form $t_{i}=g \cdot t_{j}$, where $i \neq j$ and $g \in \operatorname{Comm}(\Gamma)$.

Theorem 9. With the notation (and assumption $V(\mathbb{C}) \cap \mathbb{R}^{n} \neq$ $\emptyset)$ as above, suppose that $t_{1}, \ldots, t_{n}$ are $\Gamma$-geodesically independent. Then the $3 n$ functions

$$
j_{\Gamma}\left(t_{1}\right), j_{\Gamma}^{\prime}\left(t_{1}\right), j_{\Gamma}^{\prime \prime}\left(t_{1}\right), \ldots, j_{\Gamma}\left(t_{n}\right), j_{\Gamma}^{\prime}\left(t_{n}\right), j_{\Gamma}^{\prime \prime}\left(t_{n}\right)
$$

(defined locally) on $V(\mathbb{C})$ are algebraically independent over $\mathbb{C}(V)$.

As mentioned earlier, J. Pila [Pil13] had already proved the result for $P S L_{2}(\mathbb{Z})$. J. Freitag and T. Scanlon [FS18] established the same for arithmetic subgroups of $P S L_{2}(\mathbb{Z})$. The Ax-Lindemann-Weierstrass theorem (mostly without derivatives) has also been proved by various authors in the more general context of Shimura varieties. The work in [CFN20] differs from all the above in that it does not use a tool called o-minimality (originating in model theory) and also tackles the nonarithmetic groups as well as the derivatives of the functions all at once.

\section{Beyond $D C F_{0}$}

We end by saying a few words about the partial differential and the difference equations settings. We denote by $D C F_{0, m}$ the theory of differentially closed fields of charateristic 0 with $m$ commuting derivations (partial context) and by $A C F A$ the theory of algebraically closed fields with a generic automorphism (difference context). The theory $D C F_{0, m}$ is also $\omega$-stable and has quantifier elimination. However, strongly minimal sets do not fully capture the complexity of all definable sets. There are so-called infinite rank regular types ${ }^{3}$ that do so. The trichotomy theorem is yet to be fully established in that setting. On the other hand, $A C F A$ is not $\omega$-stable but is rather a so-called simple theory (characterized by existence of a good notion of independence). Furthermore, although definable sets are still given by simple enough formulas, $A C F A$ does not have full quantifier elimination. A version of the trichotomy theorem does hold in that setting and the study of $A C F A$ has been very successfully used to obtain new results in number theory and algebraic dynamics.

However in both cases, except for a few examples, applications to the study of classical equations is yet to be undertaken. There are obvious candidates that would mirror the situation of $D C F_{0}$. In $D C F_{0, m}$, tackling the generalized Schwarzian equations for uniformizers for Shimura varieties is of great interest. In $A C F A$, proving that the $q$ Painlevé equations are rank 1 is a challenge. These difference equations are discrete analogues of the classical Painlevé equations. In fact, in many real world problems, the Painlevé equations arise from a limiting process, starting with the q-Painlevé equations. We expect that, as with $D C F_{0}$, important model-theoretic questions about the structure of definable sets can be formed and answered by studying these concrete differential and difference equations.

\footnotetext{
${ }^{3}$ One such infinite rank regular type also exists for $D C F_{0}$. However the finite rank part of the theory is where most of the complexity lies.
} 
ACKNOWLEDGMENT. The author thanks the anonymous referees for their valuable comments and suggestions.

\section{References}

[Blu69] Lenore Carol Blum, Generalized algebraic theories: A model theoretic approach, ProQuest LLC, Ann Arbor, MI, 1969. Thesis (Ph.D.)-Massachusetts Institute of Technology. MR2939925

[Bui92] Alexandru Buium, Differential algebraic groups of finite dimension, Lecture Notes in Mathematics, vol. 1506, Springer-Verlag, Berlin, 1992. MR1176753

[CFN20] Guy Casale, James Freitag, and Joel Nagloo, AxLindemann-Weierstrass with derivatives and the genus 0 Fuchsian groups, Ann. of Math. (2) 192 (2020), no. 3, 721-765, DOI 10.4007/annals.2020.192.3.2 MR4172620

[FW15] Peter J. Forrester and Nicholas S. Witte, Painlevé II in random matrix theory and related fields, Constr. Approx. 41 (2015), no. 3, 589-613, DOI 10.1007/s00365-014-9243-5. MR3346720

[FM17] James Freitag and Rahim Moosa, Finiteness theorems on hypersurfaces in partial differential-algebraic geometry, Adv. Math. 314 (2017), 726-755, DOI 10.1016/j.aim.2017.04.008 MR3658729

[FS18] James Freitag and Thomas Scanlon, Strong minimality and the j-function, J. Eur. Math. Soc. (JEMS) 20 (2018), no. 1, 119-136, DOI 10.4171/JEMS/761 MR3743238

[Hru95] E. Hrushovski, ODEs of order 1 and a generalization of a theorem of jouanolou, Unpublished preprint (1995).

[HS94] E. Hrushovski and Z. Sokolovic, Strongly minimal sets in differentially closed fields, Unpublished preprint (1994).

[Kol73] E. R. Kolchin, Differential algebra and algebraic groups, Pure and Applied Mathematics, vol. 54, Academic Press, New York-London, 1973. MR0568864

[MMP96] D. Marker, M. Messmer, and A. Pillay, Model theory of fields, Lecture Notes in Logic, vol. 5, Springer-Verlag, Berlin, 1996. MR1477154

[Mar02] David Marker, Model theory: An introduction, Graduate Texts in Mathematics, vol. 217, Springer-Verlag, New York, 2002. MR1924282

[Nag20] Joel Nagloo, Algebraic independence of generic painlevé transcendents: PIII and PVI, Bull. Lond. Math. Soc. 52 (2020), no. 1, 100-108.

[NP14] Joel Nagloo and Anand Pillay, On the algebraic independence of generic Painlevé transcendents, Compos. Math. 150 (2014), no. 4, 668-678, DOI 10.1112/S0010437X13007525. MR3200672

[NP17] Joel Nagloo and Anand Pillay, On algebraic relations between solutions of a generic Painlevé equation, J. Reine Angew. Math. 726 (2017), 1-27, DOI 10.1515/crelle-20140082. MR3641651

[Nis04] Keiji Nishioka, Algebraic independence of Painlevé first transcendents, Funkcial. Ekvac. 47 (2004), no. 2, 351-360, DOI 10.1619/fesi.47.351 MR2108680

[Oka99] Kazuo Okamoto, The Hamiltonians associated to the Painlevé equations, The Painlevé property, CRM Ser.
Math. Phys., Springer, New York, 1999, pp. 735-787. MR1714704

[Pil13] Jonathan Pila, Modular Ax-Lindemann-Weierstrass with derivatives, Notre Dame J. Form. Log. 54 (2013), no. 3-4, 553-565, DOI 10.1215/00294527-2143853. MR3091671

[Pil02] Anand Pillay, Differential fields, Lectures on algebraic model theory, Fields Inst. Monogr., vol. 15, Amer. Math. Soc., Providence, RI, 2002, pp. 1-45, DOI 10.2178/isl/1190150158 MR1873593

[PZ03] Anand Pillay and Martin Ziegler, Jet spaces of varieties over differential and difference fields, Selecta Math. (N.S.) 9 (2003), no. 4, 579-599, DOI 10.1007/s00029-003-0339-1 MR2031753

[Rob59] Abraham Robinson, On the concept of a differentially closed field, Bull. Res. Council Israel Sect. F 8F (1959), 113128 (1959). MR125016

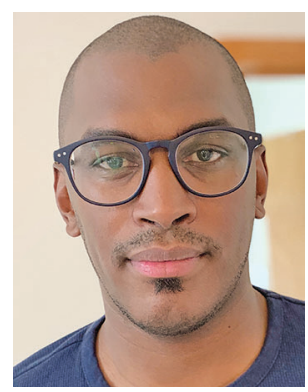

Joel Nagloo

Credits

All images in article are courtesy of Joel Nagloo.

Author photo is courtesy of Sharonne Caullychurn. 